\title{
Acute septic arthritis of the hip joint with abscess formation; a rare presentation of melioidosis
}

\author{
De Silva CM${ }^{1}$, Fonseka $\mathrm{CL}^{1,2}$, Singhapura SDAL ${ }^{1}$, Hewawithana $\mathrm{JS}^{1}$, Piyasiri DLB ${ }^{3}$, Lekamwasam $\mathrm{S}^{1,2}$ \\ ${ }^{1}$ University Medical Unit, Teaching Hospital Karapitiya, Sri Lanka. \\ ${ }^{2}$ Department of Internal Medicine, Faculty of Medicine, University of Ruhuna, Galle, Sri Lanka. \\ ${ }^{3}$ Department of Microbiology, Teaching Hospital Karapitiya, Sri Lanka.
}

Correspondence: Dr. CM De Silva

e-mail: charithamunasinghe@gmail.com

https://orcid.org/0000-0003-1745-0991

Submitted on 05.02.2020 and accepted for publication on 14.09.2020

\section{Introduction}

Melioidosis is an infectious disease caused by Burkholderia pseudomallei, a saprophytic gramnegative bacillus acquired by inhalation or inoculation through contaminated soil or water. Surface water and mud/ soil exposure especially occupational, is thought to be a major risk factor for this disease. South East Asia and Northern Australia have the highest incidence of melioidosis (1). However, India, South Pacific, Africa and the Middle East also have reported cases of melioidosis. Although Sri Lanka is not considered as an endemic country for melioidosis, it lies in the melioidosis belt, and an increasing number of cases have been reported recently (2). Although, from 1927 to 2005 only three cases had been reported in Sri Lanka, between 2006 and March 2017, 250 culture-positive cases of melioidosis and additionally over one hundred cases were diagnosed and treated as melioidosis on the basis of high antibody titres detected with improved surveillance and awareness (2). Annual incidence rates in Sri Lanka describe two distinct peaks correlating with monsoon seasons which probably indicates an increased risk of inhalation secondary to rainfall (1). Currently, there is a significant improvement in awareness and laboratory facilities which has strengthened the early diagnosis of melioidosis, thereby facilitating the commencement of appropriate antibiotic therapy to reduce the mortality and morbidity.
Melioidosis has varied clinical presentations. The presentation of melioidosis ranges from localised abscesses in skin, soft tissues and in lungs, liver or spleen to disseminated infection with fulminant septicemia (3-5). Diabetes mellitus, chronic kidney disease, liver disease and alcoholism found to be risk factors for severe infection with poor outcomes (6). Paddy and livestock farmers \& cultivators, construction workers, military personnel, and even those who indulge in gardening with occupational exposure to mud and surface water have a risk of acquiring the infection. Diagnosis lies in direct isolation of the causative bacterium from the clinical specimens (blood, urine, sputum, pus or skin-lesion sample) which is the gold standard for the definitive diagnosis. Serologic testing alone in the absence of positive cultures is inadequate for confirming the diagnosis, since in endemic regions background sero-positivity rate can be as high as 50\% $(4,6)$.

Septic arthritis and osteomyelitis are rare but recognised presentations of melioidosis (6-10). Haematogenous dissemination of disease from a focus of infection elsewhere in the body with spread to bone or joints secondarily is thought to be the mode of transmission. However, septic arthritis and osteomyelitis could be infrequent primary presenting manifestations of melioidosis. We describe a rare case of melioidosis presenting as a septic arthritis of the left hip with abscess formation without any other identifiable focus of sepsis. 


\section{Case presentation}

A 58-year-old carpenter, having type 2 diabetes mellitus for last five years presented with fever, left hip joint pain, swelling with difficulty in walking for two weeks duration. Initially, the patient complained of a low-grade fever with a left hip pain which appeared a week after the onset of fever. Gradually, his hip movements became more painful and restricted which left him confined to bed. He was initially admitted to a local hospital where he was treated as septic mono-arthritis of left hip joint with intravenous flucloxacillin without apparent resolution, hence he was transferred to a tertiary care hospital for specialised management.

On admission, the patient was found to be febrile $\left(40^{\circ} \mathrm{C}\right)$ and had significant pain in his left hip joint making it completely immobile. He was not pale and had no lymphadenopathy. He was tachycardic with pulse rate of $102 / \mathrm{min}$ and blood pressure was 120/70 mmHg. Cardiac, respiratory and abdominal examinations were unremarkable. Left hip joint was tender with limitation of all movements. Other joints were normal and there were no signs of psoas muscle irritation.

His haematological investigations revealed a neutrophil leukocytosis (WBC - $11.67 \times 10^{3} / \mu \mathrm{L} ; \mathrm{N}$ $78 \%$ ) and elevated inflammatory markers (C-RP $145 \mathrm{mg} / \mathrm{dL}$; ESR - $135 \mathrm{~mm}^{\text {st }}$ hour). His liver and renal functions and chest radiograph were normal and repeated blood cultures failed to grow an organism. Radiographs of left hip joint showed some reduction in joint space but were otherwise normal. An ultrasound scan of abdomen, pelvis and left hip joint was performed and it revealed a $5 \times 3 \mathrm{~cm}$ abscess around hip joint with significant joint effusion but did not reveal abscesses elsewhere.

An ultrasonically guided aspiration of left hip joint area revealed thick yellowish pus which did not reveal any organisms or abnormal cells on microscopy. However, the culture of this fluid revealed a growth of a gram-negative bacillus which was resistant to aminoglycosides and polymyxin B which later was identified as Burkholderia pseudomallei. His serum antibody titre for melioidosis became positive (1: 640 by Indirect Haemagglutination Assay) on two occasions. He was commenced on Intravenous meropenam $1 \mathrm{~g} 8 \mathrm{~h}$ hourly, oral trimethoprim-sulfamethoxazole 1440 mg twice daily and with the help of orthopaedic team hip joint arthrotomy and abscess drainage was performed twice. He showed excellent response to therapy and graded physiotherapy and mobilisation was commenced. His fever and C-RP started to respond within $72 \mathrm{hrs}$ of antibiotics and inflammatory markers showed a remarkable response. He was able to walk with minimal pain within 10 days of appropriate antibiotics and rehabilitation and was discharged from hospital after 21 days, on oral trimethoprimsulfamethoxazole and doxycycline eradication therapy. He fully recovered within next three months without any relapses.

\section{Discussion}

Melioidosis is an important emerging infection in Sri Lanka which has a wide range of clinical manifestations. Our patient had a history of diabetes mellitus with regular consumption of alcohol which were described as predisposing factors for development of melioidosis. Being a carpenter with frequent visits to forests for wood cutting would have increased the risk of exposure to soil.

Septic arthritis is a rare, but well recognised and reported manifestation of melioidosis (11). In addition to the primary joint related disease presentations, some patients can develop secondary osteomyelitis or septic arthritis over the course of illness as a part of disseminated sepsis due to melioidosis with a primary focus elsewhere such as pneumonia, particularly in endemic areas with the presence of aforementioned risk factors. In the clinical setting, differentiation between septic arthritis due to Burkholderia pseudomallei and of other common pathogens like Staphylococcus aureus or Streptococcus spp. can be extremely challenging. Both involves large joints, such as knee, ankle, elbow, hip and shoulder (10). However, in melioidosis knee is the commonest joint to be involved (6). Our patient had isolated hip joint involvement, where there is limited reported evidence of this presentation even from Northern Australia and Thailand, where melioidosis is highly endemic. 
Bone and joint involvement due to $B$. pseudomalle $i$ ranges from acute septic arthritis to chronic osteomyelitis / arthritis. Metaphyseal regions of long bones are the commonest sites of osseous lesions, along with vertebral bodies. Radiological appearances may demonstrate cortical bone erosions or cystic degenerations. Prompt diagnosis with isolation of organism from cultures with a positive/rising titer of serology is necessary to differentiate from pyogenic abscesses, tuberculosis, neoplasm, sarcoidosis and other granulomatous disease (7). Bone and joint involvement in melioidosis was identified in $7.6 \%$ of melioidosis patients in an Australian study (6); while a Thai study (11) showed a figure of $14-27 \%$ with more proximal joint involvement (6).

So far, there are only few published case reports describes cases of isolated septic arthritis of a hip joint following melioidosis $(12,13)$, where Rodrigo et al described a case showing septic arthritis of hip with intramuscular abscesses around the hip and proximal femur with cultures obtained from the aspiration of abscess being positive for Pseudomonas spp. which mimicked melioidosis. Culture of the hip joint aspiration did not grow anything in this patient (13). Our patient's culture of the aspirated joint fluid became positive for a gram negative organism. This educated us that in the appropriate clinical setting, culture reports of gram-negative organism or Pseudomonas spp. may be a clue that we should probe further to identify the organism of melioidosis either by supportive serology or polymerase chain reaction (PCR) and the discussions with the microbiology team will be important so that a closer assessment at the cultures to identify the organism can be made with further subtyping of organism in reference laboratories. Weerasinghe et al., have described another case where the cultures have not become positive although the IHA titres have been very high $(10,240)$ where the specific treatment directed for melioidosis has led to a complete recovery (12). In our case, we observed a culture poitivity in the ultrasonically guided aspiration fluid from left hip joint to diagnose melioidosis.

With further emergence of melioidosis we would come across more patients with primary osteomyelitis and septic arthritis warranting a high degree of clinical suspicion for accurate diagnosis.

Identification of the organism correctly with its characteristic antibiotic sensitivity pattern and the colony morphology is imperative at the microbiology laboratory with confirmation by serology or latex agglutination and PCR later. Also, presence of abscess formation with the septic arthritis could be a pointer of melioidosis over other organisms. A high degree of clinical and microbiological suspicion and awareness of melioidosis are important for institutions within endemic areas. Prompt commencement of appropriate antibiotics, thorough and repeated debridement of infected bones and joint washouts where indicated, and early and graded physiotherapy and mobilisation are the cornerstones of successful outcome (7-9). Our report adds to the literature of an important manifestation of melioidosis and highlights the rare involvement of an isolated joint with excellent recovery with prompt and appropriate antibiotic treatment and surgical intervention.

Informed consent was obtained from the patient for publication of this case report.

\section{Conclusions}

Acute septic arthritis of the hip due to melioidosis did not have characteristic features to differentiate with other causes of pyogenic septic arthritis, posing a difficulty in suspecting melioidosis unless proper microbiological investigations are performed. Therefore, melioidosis needs to be actively investigated performing cultures and serology, especially in the presence of risk factors such as diabetes etc. This case report has been presented to raise the awareness of an unusual presentation of melioidosis.

\section{Acknowledgements}

Department of Microbiology, Faculty of Medicine, University of Colombo for providing melioidosis IHA titers. 


\section{References}

1. Corea EM, De Silva AD, Thevanesam V. Melioidosis in Sri Lanka. Tropical Medicine and Infectious Disease. 2018;3(1): 22 .

2. Corea EM, Merritt AJ, Ler YH, Thevanesam V, Inglis TJJ. Sri Lankan national melioidosis surveillance program uncovers a nationwide distribution of invasive melioidosis Am. J. Trop. Med. Hyg. 2016; 94: 292-298.

3. White NJ. Melioidosis. The Lancet. 2003;361: 1715-1722.

4. Leelarasamee A, Bovornkitti S. Melioidosis: Review and update. Reviews of Infectious Diseases. 1989; 2: 413-425.

5. Jayasekara K, Perera S, Wijesundere A. Fatal Burkholderia pseudomallei septicemia. Ceylon Medical Journal. 2006; 51: 69-70.

6. Currie BJ, Ward L, Cheng AC. The epidemiology and clinical spectrum of melioidosis: 540 cases from the 20 year Darwin prospective study. PloS Negl Trop Dis. 2010; 4(11): e900. DOI: https://doi.org/10.1371/ journal.pntd.0000900

7.. Subhadrabandhu T, Prichasuk S, Sathapatayavongs B Localised melioidotic osteomyelitis. J Bone Jt Surg [Br]. 1995; 77-B: 445-449.

8. Popoff I, Nagamori J, Currie B. Melioidotic osteomyelitis in northern Australia. Aust NZJ Surg. 1997; 67: e692-e695. https://doi.org/10.1111/j.1445-2197.1997.tb07111.x
9. Kosuwon W, Saengnipanthkul S, Mahaisavariya B, Laupattarakasem W. Musculoskeletal melioidosis. JBJS. 1993; 75(12): 1811-1815.

10. Teparrakkul Á, Tsai JJ, Chierakul W, Gerstenmaier JF, Wacharaprechasgu T, Piyaphanee W, Limmathurotsakul D, Chaowagul W, Day NP, Peacock SJ. Rheumatological manifestations in patients with melioidosis. Southeast Asian Journal of Tropical Medicine and Public Health. 2008; 39(4): 649-655.

11. Kosuwon W, Taimglang T, Sirichativapee W, Jeeravipoolvarn P. Melioidotic septic arthritis and its risk factors. JBJS. 2003; 85(6): 1058-1061.

12. Weerasinghe NP, Herath HM, Liyanage TM. Isolated septic arthritis of hip joint: a rare presentation of melioidosis. A case report. BMC Research Notes. 2018; 11(1): 50. DOI: http://doi.org/10.1186/s13104-018-31716.

13. Rodrigo KMD, Premaratna R, De Silva HJ, Corea E. Melioidosis as a cause of femoral osteomyelitis and multifocal intramuscular abscess around the hip joint in a farmer: a case report. Sri Lankan Journal of Infectious Diseases. 2013; 3(1):50-54. DOI: http://dx.doi.org/ 10.4038/sljid.v3i1.4655. 